\title{
Anisotropic exchange interaction induced by a single photon in semiconductor microcavities
}

\author{
G. Chiappe, ${ }^{1,2}$ J. Fernández-Rossier, ${ }^{1}$ E. Louis, ${ }^{1}$ and E. V. Anda $^{3}$ \\ ${ }^{1}$ Departamento de Física Aplicada, Instituto Universitario de Materiales and Unidad Asociada del CSIC, Universidad de Alicante, \\ San Vicente del Raspeig, Alicante 03690, Spain \\ ${ }^{2}$ Departamento de Física J.J. Giambiagi, Facultad de Ciencias Exactas, Universidad de Buenos Aires, Ciudad Universitaria, \\ 1428 Buenos Aires, Argentina \\ ${ }^{3}$ Departamento de Física, Pontificia Universidade Católica do Rio de Janeiro (PUC-Rio), 22452-970, Caixa Postal 38071, \\ Rio de Janeiro, Brazil
}

(Received 23 May 2005; revised manuscript received 20 September 2005; published 8 December 2005)

\begin{abstract}
We investigate coupling of localized spins in a semiconductor quantum dot embedded in a microcavity. The lowest cavity mode and the quantum dot exciton are coupled and close in energy, forming a polariton. The fermions forming the exciton interact with localized spins via exchange. Exact diagonalization of a Hamiltonian in which photons, spins, and excitons are treated quantum mechanically shows that a single polariton induces a sizable indirect anisotropic exchange interaction between spins. At sufficiently low temperatures strong ferromagnetic correlations show up without an appreciable increase in exciton population. In the case of a $(\mathrm{Cd}, \mathrm{Mn}) \mathrm{Te}$ quantum dot, Mn-Mn ferromagnetic coupling is still significant at $1 \mathrm{~K}$ : spin-spin correlation around 3 for exciton occupation smaller than 0.3 . We find that the interaction mediated by photon-polaritons is 10 times stronger than the one induced by a classical field for equal Rabi splitting.
\end{abstract}

DOI: 10.1103/PhysRevB.72.245311

PACS number(s): 73.63.Fg, 71.36.+c, 71.35.-y, 71.15.Mb

Control of exchange interactions in solid-state environments has become a strategic target in the development of spintronics and quantum computing. ${ }^{1-3}$ Artificial control of direct exchange interactions, which occur at length scales of one lattice spacing, is hardly possible with current day technologies. In contrast, indirect exchange interactions (IEI's) of spins sitting several nanometers away can be tuned, taking advantage of the optical and electrical manipulation of the intermediate fermions afforded in semiconducting hosts. ${ }^{1,2,4-8}$ The local spins could be provided by nuclei, electrons bound to donors, ${ }^{4,6}$ or $d$ electrons of magnetic impurities. ${ }^{7,8}$ Laser-induced IEI's in semiconductors can be tuned by changing the laser frequency, intensity, and polarization. Above gap excitation ${ }^{6,9}$ provides real carriers that mediate "RKKY-like" exchange. Below gap excitation induces an optical coherence between conduction and valence bands able to mediate exchange interactions between localized spins. ${ }^{4,8}$ The strength of the "optical RKKY" (ORKKY) exchange interaction is determined by the intensity of the laser, proportional to the square of the Rabi energy $\Omega$, and by the detuning $\delta=E_{g}-\omega_{L}$ between the semiconductor gap and the laser frequency. ${ }^{4}$ When $\delta>\Omega$, the laser-matter coupling can be treated in perturbation theory, ${ }^{4}$ which gives a coupling strength $\propto \Omega^{2} / \delta^{3}$.

Motivated by recent experimental results, we propose a system where the optical exchange interaction is greatly enhanced: a semiconductor micropillar cylindric cavity, ${ }^{10-12}$ made of CdTe with inclusions of CdTe quantum dots ${ }^{13,14}$ doped with a few Mn atoms. The Mn spins are exchange coupled to both electrons and holes confined in the quantum dots. ${ }^{13,14}$ Recent experiments show that it is possible to fabricate dots with a single $\mathrm{Mn}$ atom inside. ${ }^{14}$ In turn, quantum-dot electron-hole pairs (excitons) are coupled to the confined photon field. Pillar microcavities based on CdMnTe/CdMgTe heterostructures have been fabricated ${ }^{11}$ featuring strong coupling between two-dimensional (2D) ex- citons with OD photons with a Rabi energy as high as $16 \mathrm{meV}$, which can hardly be obtained with cw lasers. The strong-coupling regime between III-V quantum dot excitons and $0 \mathrm{D}$ photons in $\mathrm{OD}$ cavities has been recently reported, with a Rabi couplings as big as $\simeq 0.4 \mathrm{meV} .{ }^{12}$ On the other side, there is experimental ${ }^{9}$ and theoretical ${ }^{7}$ evidence that the effective Mn-Mn interactions mediated by fermions with full 3D confinement are strongly enhanced compared with the case of bulk fermions.

The exact solution of Hamiltonian, considering all degrees of freedom fully quantum mechanically, shows that confinement of both light and intermediate fermions yields an enhancement of the ORKKY interaction. For cavity modes below the exciton threshold $(\delta>0)$ and at sufficiently low temperatures, strong ferromagnetic coupling shows up without a significant increase in exciton density. Exchange coupling is so big that calculations for a $(\mathrm{Cd}, \mathrm{Mn}) \mathrm{Te}$ quantum dot indicate that a single polariton can induce ferromagnetic coupling between two localized spins at temperatures of $1 \mathrm{~K}$.

The model. The dot that confines conduction-band electrons (creation operator $c_{\sigma}^{\dagger}$, where $\sigma$ denotes the spin) and valence-band electrons (creation operator $v_{\sigma}^{\dagger}$ ) (Ref. 15) has intraband-level spacing larger than all the other interband energy scales, so that we only keep the lowest orbital level in each band, $\epsilon_{c}$ and $\epsilon_{v}$. These levels have a twofold spin degeneracy. Their orbital wave functions are $\psi_{c}(\vec{r})$ and $\psi_{v}(\vec{r})$, respectively. The electric field of the lowest cavity mode lies in the plane perpendicular to the axis of the cylinder. So there are two degenerate cavity modes associated with the two possible polarization states in that plane. Their energy $\hbar \omega$ is close to the quantum-dot band gap. We choose circularly polarized cavity modes and, after canonical quantization, the corresponding photon creation operator is denoted by $b_{\lambda}^{\dagger}$, where $\lambda=L, R$. We assume a cavity with an infinite quality factor. ${ }^{16}$ 
The Hamiltonian we consider here has three terms, $\mathcal{H}=\mathcal{H}_{0}+\mathcal{H}_{g}+\mathcal{H}_{J}$. The first describes the Hamiltonian for decoupled cavity photons and electrons,

$$
\mathcal{H}_{0}=\sum_{\lambda} \hbar \omega b_{\lambda}^{\dagger} b_{\lambda}+\sum_{\sigma}\left[\epsilon_{v} v_{\sigma}^{\dagger} v_{\sigma}+\epsilon_{c} c_{\sigma}^{\dagger} c_{\sigma}\right]
$$

All results presented below correspond to $\epsilon_{v}=0$ and $\epsilon_{c}=E_{g}$, where $E_{g}$ is the semiconductor band gap.

The light-matter coupling Hamiltonian is

$$
\mathcal{H}_{g}=g \sum_{\lambda, \sigma}\left(b_{\lambda}^{\dagger}+b_{\lambda}\right)\left[c_{\sigma}^{\dagger} v_{\sigma}+v_{\sigma}^{\dagger} c_{\sigma}\right] \delta_{\lambda, \sigma}
$$

where we have assumed that the hole is purely heavy, which leads to the standard spin selective coupling ${ }^{4,8,17}$ that associates photon polarization and fermion spin degrees of freedom. This coupling breaks spin rotational invariance and privileges the axis of the cavity, $\hat{z}$. The value of $g$ depends on the amplitude of the cavity mode in the dot and plays the same role than the Rabi energy $\Omega$ in the case of a photoexcited semiconductor. ${ }^{4,8}$ We take $g=5 \mathrm{meV}$ which is within the range of Rabi splittings reported in the literature for CdTe nanopillars (larger values have been reported experimentally $\left.{ }^{11}\right)$. Another key quantity is the detuning $\delta=E_{g}-\hbar \omega$. For $\mathcal{N}=1$ excitation the ground-state manifold is mainly photonic for $\delta \gg 0$, mainly excitonic for $\delta \ll 0$, and it is a compensated mixture around $\delta=0$.

The exchange interaction between the fermions and the spin $M=5 / 2$ of the Mn impurities reads

$$
\mathcal{H}_{J}=\sum_{I, f} J_{f} \vec{M}_{I} \cdot \vec{S}_{f}\left(\vec{x}_{I}\right)
$$

where $\vec{S}_{f}\left(\vec{r}_{I}\right)$ stands for local spin density of the $f=v, c$ electron and $\vec{M}_{I}$ is the Mn spin located at $\vec{r}_{I}$. The electron spin density is

$$
\vec{S}_{f}\left(\vec{r}_{I}\right)=\frac{1}{2}\left|\psi_{f}\left(\vec{r}_{I}\right)\right|^{2} c_{\sigma}^{\dagger} c_{\sigma^{\prime}} \vec{\tau}_{\sigma, \sigma^{\prime}},
$$

where $\vec{\tau}$ are the Pauli matrices. The strength of the interaction depends both on the exchange constant of the material $J_{f}$ and on the localization degree of the carrier, $\left|\psi_{f}\left(\vec{r}_{I}\right)\right|^{2}$. We consider a hard-wall quantum dot, with lateral dimensions $L \simeq 10 \mathrm{~nm}$ and total volume $\simeq 1200 \mathrm{~nm}^{3}$. In such a dot, a realistic value for the exchange interaction between a conduction- (valence-) band electron and a Mn spin is $J_{c}=-0.1 \mathrm{meV}\left(J_{v}=-0.5 \mathrm{meV}\right)$. These values were used in all calculations discussed hereafter.

The band gap in $(\mathrm{Cd}, \mathrm{Mn}) \mathrm{Te}$ is the largest energy scale $\left(E_{g} \simeq 2 \mathrm{eV}\right)$. Therefore the effect of the terms that do not conserve the number of excitons plus photons is negligible and they can safely be removed. This permits to work in subspaces with $\mathcal{N}$ excitations. Here we consider the coupling between two $\mathrm{Mn}$ impurities in the presence of $\mathcal{N}=1$ polaritons. We define a spin-spin correlation

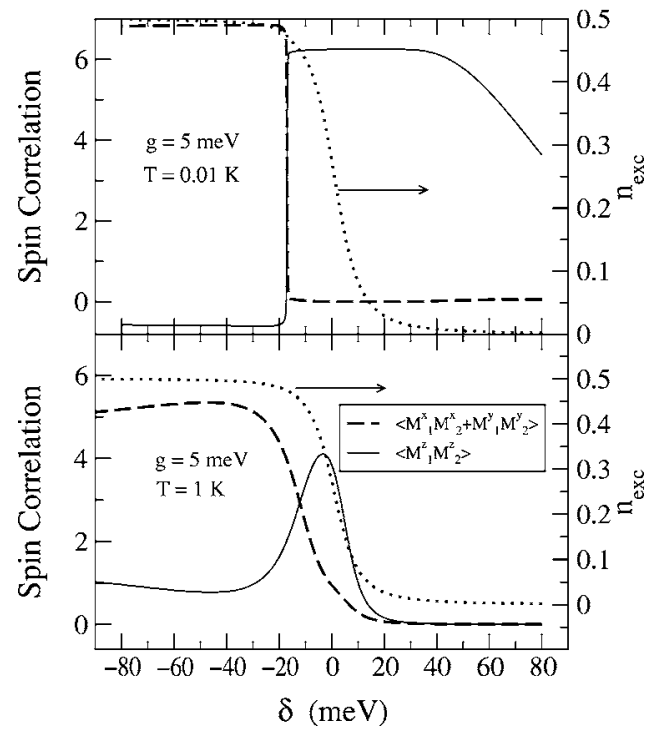

FIG. 1. Spin correlation functions and exciton occupation number versus the detuning parameter $\delta$, for spin-5/2 impurities. $g=5 \mathrm{meV}$ and $T=0.01 \mathrm{~K}$ (upper) and $1 \mathrm{~K}$ (lower).

$$
\left\langle\vec{M}_{1} \cdot \vec{M}_{2}\right\rangle=\frac{1}{Z} \sum_{i}\left\langle\Phi_{i}\left|\vec{M}_{1} \cdot \vec{M}_{2}\right| \Phi_{i}\right\rangle \mathrm{e}^{-E_{i} / k_{B} T}
$$

where $Z$ is the partition function and the sum runs over the eigenstates $\Psi_{i}$ of the Hamiltonian with energies $E_{i}$.

Results for quantized cavity modes. Figure 1 depicts results for out-of-plane $\left\langle M_{1}^{z} M_{2}^{z}\right\rangle$ and in-plane $\left\langle M_{1}^{x} M_{2}^{x}+M_{1}^{y} M_{2}^{y}\right\rangle$ spin-spin correlations and the exciton occupation number $\left(n_{\text {exc }}\right)$ versus $\delta$, for $g=5 \mathrm{meV}, T=0.01$, and $1 \mathrm{~K}$. We highlight the following results: (i) strong spin-spin correlations may show up for $\delta \gg g>0$ and negligible $n_{\text {exc }}$, (ii) the exchange interaction is anisotropic and the correlation shifts from out of plane to in plane as $\delta$ goes through zero, and (iii) the coupling may be antiferromagnetic in one of the spin components, while being ferromagnetic for the spin as a whole.

Analysis of the many-body spectrum of the Hamiltonian, shown in Fig. 2 for the case of two spin-1/2 spins coupled to

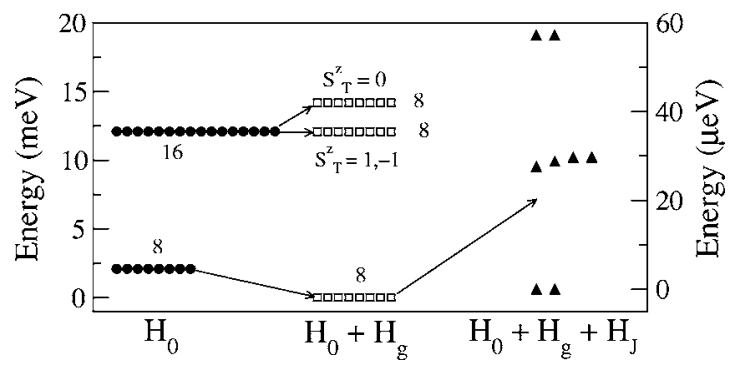

FIG. 2. Energy levels for spin-1/2 impurities calculated either without interactions (left) incorporating light-matter coupling (middle) or with the full interacting Hamiltonian (right). The energy scale in the first two cases (third case) is given on the left (right) vertical axis. The degeneracy of each state is also indicated. All results correspond to $\delta=10 \mathrm{meV}$ and $g=5 \mathrm{meV}$. Energies referred to the ground state of the full Hamiltonian in each case. 
one exciton polariton, helps to understand the results of Fig. 1. Hereafter we refer to photonic (excitonic) configurations to those having one (no) photon and no (one) exciton. For $g=0$ and no exchange interaction, the ground state is eightfold degenerate: two photon configurations ( $L$ or $R$ ), no exciton, and four impurity configurations (see Fig. 2 left). Switching light-matter coupling does not remove the degeneracy of the ground state. This is so because each configuration with a $R(L)$ photon couples only to one exciton configuration having a conduction-band electron with spin up (down); see Fig. 2, middle. In these states $S_{T}^{z}=S_{v}^{z}+S_{c}^{z}=0$. States with $S_{T}^{z}= \pm 1$ do not couple with light, and its energy is not modified. The way exchange coupling removes this degeneracy (without mixing degenerate states) is better understood by rewriting $\mathcal{H}_{J}$ as

$$
\mathcal{H}_{J}=J_{v}\left(\vec{M}_{1}+\vec{M}_{2}\right) \cdot\left(\vec{S}_{v}+\vec{S}_{c}\right)+\left(J_{c}-J_{v}\right)\left(\vec{M}_{1}+\vec{M}_{2}\right) \cdot \vec{S}_{c} .
$$

Degeneracy is lifted in first order as diagonal elements from the second term in $\mathcal{H}_{J}$ give nonzero contributions. As $J_{c}-J_{v}>0$, the two states in which the two impurities have parallel spins antiferromagnetically (ferromagnetically) coupled with the photon and conduction band electron lower (increase) their energy (see Fig. 2, right). The configurations having the two impurities with opposite spins do not change their energy in first order (higher-order corrections modify slightly this result). Then, the ground state is no longer eightfold degenerate but rather twofold: two photonic configurations with impurities having spins that are parallel to each other and opposite to the photon polarization, slightly mixed with one excitonic configuration each. Consequently, the correlation is totally out of plane. Exactly the same happens for $M_{i}=5 / 2$ spins, except for the fact that now there are 36 local spin configurations and that the resulting exchange splittings, which scale with $M_{i}$, are larger. Thus, the same physics underlies the results of Fig. 1.

The changes in anisotropy and the antiferromagnetic correlation that show up as $\delta$ becomes negative can be explained as follows. For negative detuning the ground state is composed of all excitonic configurations with $S_{T}^{z}=0$, plus a small photonic contribution. Excitonic configurations with $S_{T}^{z}= \pm 1$ have greater energy as they do not interact with light. But as a consequence of the ferromagnetic coupling between the impurities and exciton, energy is minimized if the exciton spin is not zero. Thus, it must lie in the $x-y$ plane and the spin of the impurities must also lie mostly in that plane. This favors in-plane ferromagnetic correlation between impurities, plus a small out-of-plane correlation whose character (ferromagnetic or antiferromagnetic) crucially depends on detuning and temperature (for very large detuning or very large temperatures, isotropy is recovered).

Figure 3 shows the spin-spin correlation versus $n_{\text {exc }}$ (varied by changing $g$ ) for several temperatures and $\delta=10 \mathrm{meV}$. At temperatures $T \leqslant 0.1 \mathrm{~K}$, the spin-spin correlation has a nonmonotonous relation with the exciton density, which reflects the failure of perturbation theory in $g$ when $n_{\text {exc }} \simeq 0.2$. In addition, at $T=1 \mathrm{~K}$, significant spin-spin correlations are obtained for $n_{\text {exc }}<0.3$. The spin-spin correlation monotoni-

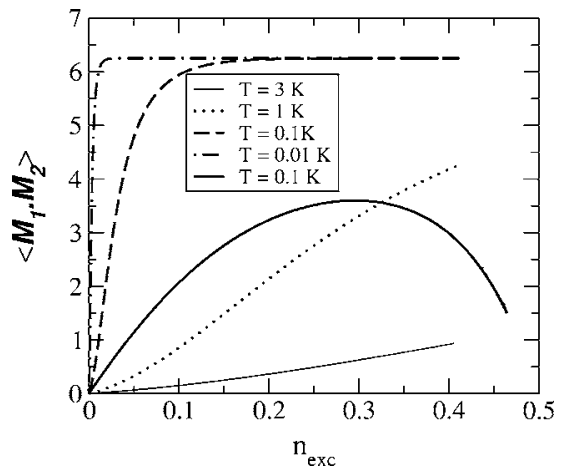

FIG. 3. Total spin-spin correlation versus exciton occupation number for spin-5/2 impurities and four temperatures. Calculations were carried out by varying $g$ and with fixed detuning $\delta=10 \mathrm{meV}$. The results derived from a classical treatement of the photon field (see text) is also shown (thick solid line).

cally increases with the exciton population in the quantum case. This is due to the fact that the first-order correction, which lifts the degeneracy of the ground state, is proportional to $n_{\mathrm{exc}}$. Figure 4 shows the in-plane and out-of-plane spin-spin correlation versus exciton population for $\delta=10 \mathrm{meV}$ and $T=0.1 \mathrm{~K}$. As could be inferred from Fig. 1 and the related discussion for this positive detuning, the spinspin correlation is mostly out of plane. The vanishing of in-plane correlation as the exciton occupation increases can be understood as follows. As excitons with $S_{z}=0$ are coupled to light, their energy increases with light-matter coupling (and, thus, with exciton occupation number), diminishing their contribution to the ground state (see also preceding paragraph).

The behavior of the spin-spin correlation versus $n_{\text {exc }}$ resembles that of correlation versus coupling $J$ in a Heisenberg Hamiltonian. In fact, in the case of two spin-1/2 impurities the spin-spin correlation $\left\langle\vec{M}_{1} \cdot \vec{M}_{2}\right\rangle$ $=(3 / 4)\left[1-\exp \left(-J / k_{B} T\right)\right] /\left[3+\exp \left(-J / k_{B} T\right)\right]$ shows a behavior similar to that of Fig. 3. Thus, we have attempted to fit

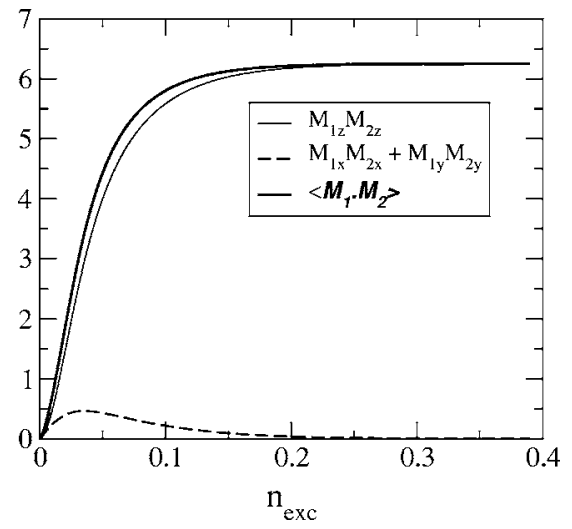

FIG. 4. In-plane and out-of-plane spin-spin correlation versus exciton occupation number for spin-5/2 impurities. Calculations were carried out by varying $g$ and with fixed detuning $\delta=10 \mathrm{meV}$. The results were obtained for $T=0.1 \mathrm{~K}$ and valence- and conduction-band exchange couplings of $J_{v}=-0.5 \mathrm{meV}$ and $J_{c}=-0.1 \mathrm{meV}$, respectively. 


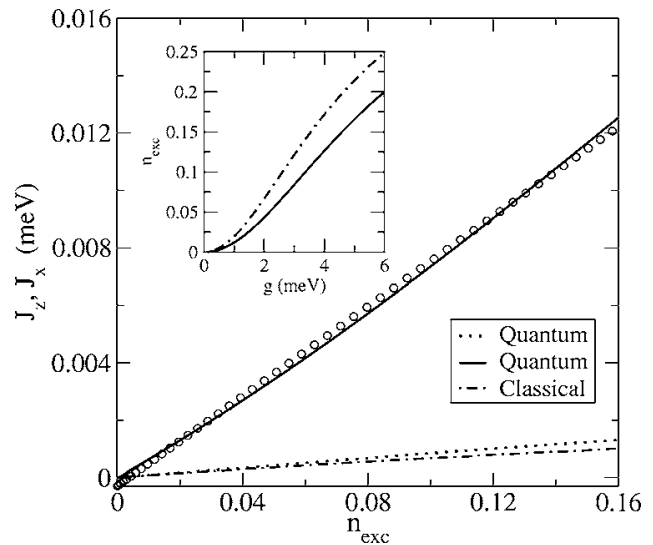

FIG. 5. $J_{x}$ (dotted line) and $J_{z}$ (solid line), parameters of the Hamiltonian of Eq. (7), fitted to give the exact spin-spin correlation, versus the exciton occupation number. Calculations were carried out by varying $g$ and with fixed detuning $\delta=10 \mathrm{meV}$. The fitting of the numerical results for $J_{z}$ by means of a straight line (circles) is also shown. Chain lines show the results obtained by treating the photon field classically (see text).

the exact results for the spin-spin correlation by means of an anisotropic Heisenberg Hamiltonian

$$
\mathcal{H}_{e f f}=-J_{x}\left(M_{1}^{x} M_{2}^{x}+M_{1}^{y} M_{2}^{y}\right)-J_{z} M_{1}^{z} M_{2}^{z} .
$$

The two parameters were fitted to give the spin-spin correlation over the temperature range $T=0-6 \mathrm{~K}$. Remarkably, it is always possible to find $J_{x}$ and $J_{z}$ such that correlations are similar to a few percent. Fittings improve significantly away from $\delta=0$. The results for $\delta=10 \mathrm{meV}$ are shown in Fig. 5. In this case $J_{z}$ is almost an order of magnitude larger than $J_{x}$. Both parameters vary linearly with $n_{\mathrm{exc}}$ (the fitting for $J_{z}$ is shown in Fig. 5).

Results in the semiclassical limit. In order to ascertain the differences between the original ORKKY proposal and this work, we consider a spin-doped dot driven by a classical electromagnetic field. For a $\mathrm{cw}$ laser and within the rotatingwave approximation, two modifications have to be introduced in the full Hamiltonian: ${ }^{8}$ (i) the quantized photon operators are removed from $\mathcal{H}_{0}$ and the semiconductor gap $E_{g}$ is replaced by $\delta$ and (ii) and the light-matter coupling of Eq. (2) is written as

$$
\mathcal{H}_{g, c l}=\sum_{\sigma} g\left[c_{\sigma}^{\dagger} v_{\sigma}+v_{\sigma}^{\dagger} c_{\sigma}\right]
$$

where we assume equal amplitudes for the two circular polarizations of the exciting classical laser. Results obtained through exact diagonalization of such a Hamiltonian are shown in Figs. 3, 5, and 6. Comparing with the full quantum solution we note that (i) the spin-spin correlation is substantially smaller (see Fig. 3), (ii) the correlation does not saturate as a function of $g$, but rather decreases at large $g$ (Fig. 3), (iii) the effective Heisenberg Hamiltonian is isotropic and has a coupling constant almost an order of magnitude smaller (Fig. 5), and (iv) $n_{\text {exc }}$ increases with $g$ at a faster pace (inset of Fig. 5).

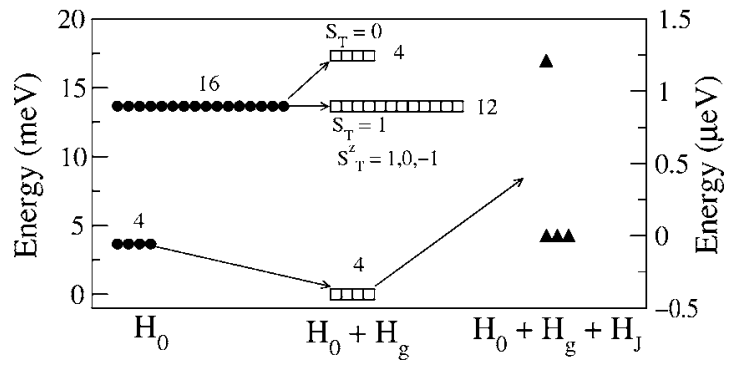

FIG. 6. Same as Fig. 2 but treating the photon field classically.

Insight into these results is gained by inspection of the Hamiltonian spectrum for the case of spin-1/2 local spins. Without interactions and for $\delta>0$ (see Fig. 6) the ground state is fourfold degenerate: four configurations of the impurities spin and no exciton (there are no photon degrees of freedom). When $g>0$, the light-matter interaction couples the bright exciton configurations the zero-exciton configurations. Since we are imposing a polarization state in the laser, there is only one bright exciton and three dark exciton states, with fourfold degeneracy due to the two underlying spins $1 / 2$. When exchange interaction is switched on, it yields no contribution to linear order in $J$. Consequently, the ground state is perturbed by the exchange interaction only to second order, leading to a much smaller splitting than in the quantum case (Fig. 6, right) and, thus, to a large reduction of the effective interaction between impurities. The behavior of the exchange splitting as a function of $g$ is governed by the exciton content of the lower polariton branch on one side and the energy splitting between the lower and upper polariton branches on the other. This results in the nonmonotonic behavior of the exchange splitting and the magnetic correlations as a function of $g$ in the semiclassical case (see Fig. 3). A related result not shown in the figures is that the effective exchange coupling is proportional to $n_{\mathrm{exc}}$ only for small values of the exciton population (as opposed to the quantum case where a linear relation between both quantities was found over a wide range; see Fig. 5). Perturbation theory correctly reproduces this linear relationship at small $n_{\text {exc }}{ }^{8}$

Summarizing, we have studied the indirect exchange interaction between two spins in a cavity-dot system with one polariton. At $T \leqslant 0.1 \mathrm{~K}$, maximum spin correlation is obtained for negligible exciton occupation numbers. The anisotropy of the exchange interaction can be tuned by varying $\delta$. In addition it is possible to fit the numerical results for the spin-spin correlation by means of an anisotropic Heisenberg Hamiltonian. We find that the quantized electromagnetic field of a single photon in a cavity mode happens to induce a signicantly larger ORKKY interaction than a classical field yielding the same Rabi energy.

We acknowledge C. Piermarocchi for fruitful discussions. G.C. and J.F.R. acknowledge financial support from the Ramón y Cajal Program (MCyT, Spain) and from CAV, Grant No. GV05/152. Partial financial support by the Spanish MCYT (Grant Nos. MAT2002-04429-C03, MAT200308109-C02-01, and FIS200402356), the Brazilian agencies FAPERJ, CAPES, and CNPq, and the Argentinian UBACYT and CONICET is gratefully acknowledged. 
${ }^{1}$ D. Loss and D. P. DiVincenzo, Phys. Rev. A 57, 120 (1998).

${ }^{2}$ A. Imamoglu, D. D. Awschalom, G. Burkard, D. P. DiVincenzo, D. Loss, M. Sherwin, and A. Small, Phys. Rev. Lett. 83, 4204 (1999).

${ }^{3}$ Semiconductor Spintronics and Quantum Computation, edited by D. D. Awschalom, D. Loss, and N. Samarth (Springer, New York, 2002).

${ }^{4}$ C. Piermarocchi, P. Chen, L. J. Sham, and D. G. Steel, Phys. Rev. Lett. 89, 167402 (2002); C. Piermarocchi and G. F. Quinteiro, Phys. Rev. B 70, 235210 (2004).

${ }^{5}$ T. Calarco, A. Datta, P. Fedichev, E. Pazy, and P. Zoller, Phys. Rev. A 68, 012310 (2003).

${ }^{6}$ J. M. Bao, A. V. Bragas, J. K. Furdyna, and R. Merlin, Nat. Mater. 2, 175 (2003).

${ }^{7}$ J. Fernández-Rossier and L. Brey, Phys. Rev. Lett. 93, 117201 (2004).

${ }^{8}$ J. Fernández-Rossier, C. Piermarocchi, P. Chen, A. H. MacDonald, and L. J. Sham, Phys. Rev. Lett. 93, 127201 (2004).

${ }^{9}$ S. Mackowski, T. Gurun, T. A. Nguyen, H. E. Jackson, L. M. Smith, G. Karczewski, and J. Kossut, Appl. Phys. Lett. 84, 3337 (2004).

${ }^{10}$ G. S. Solomon, M. Pelton, and Y. Yamamoto, Phys. Rev. Lett. 86, 3903 (2001).

${ }^{11}$ M. Obert, J. Rennert, A. Forchel, G. Bacher, R. Andre, and D. L.
S. Dang, Appl. Phys. Lett. 84, 1435 (2004).

${ }^{12}$ J. P. Reithmaier, G. Sek, A. Loffler, C. Hofmann, S. Kuhn, S. Reitzenstein, L. V. Keldysh, V. D. Kulakovskii, T. L. Reinecke, and A. Forchel, Nature (London) 432, 197 (2004); A. Badolato, K. Hennessy, M. Atatre, J. Dreiser, P. M. Petroff, and A. Imamoglu, Science 308, 1158 (2005); E. Peter, P. Senellart, D. Martrou, A. Lemaitre, J. Hours, J. M. Grard, and J. Bloch, Phys. Rev. Lett. 95, 067401 (2005).

${ }^{13}$ A. A. Maksimov, G. Bacher, A. McDonald, V. D. Kulakovskii, A. Forchel, C. R. Becker, G. Landwehr, and L. W. Molenkamp, Phys. Rev. B 62, R7767 (2000); G. Bacher, A. A. Maksimov, H. Schomig, V. D. Kulakovskii, M. K. Welsch, A. Forchel, P. S. Dorozhkin, A. V. Chernenko, S. Lee, M. Dobrowolska, and J. K. Furdyna, Phys. Rev. Lett. 89, 127201 (2002).

${ }^{14}$ L. Besombes, Y. Léger, L. Maingault, D. Ferrand, H. Mariette, and J. Cibert, Phys. Rev. Lett. 93, 207403 (2004); Y. Léger, L. Besombes, L. Maingault, D. Ferrand, and H. Mariette, ibid. 95, 047403 (2005).

${ }^{15}$ A. Barenco and M. A. Dupertuis, Phys. Rev. B 52, 2766 (1995).

${ }^{16}$ Calculations for realistic values of the quality factor $(Q$ $=100-300$; see Ref. 12) give very similar results.

${ }^{17}$ Optical Orientation, edited by F. Meier and B. P. Zakharchenya (North-Holland, New York, 1984). 